\title{
Therapeutic Efficacy of pH-Dependent Release Formulation of Mesalazine on Active Ulcerative Colitis Resistant to Time-Dependent Release Formulation: Analysis of Fecal Calprotectin Concentration
}

\author{
Kousaku Kawashima, ${ }^{1}$ Shunji Ishihara, ${ }^{1}$ Takafumi Yuki, ${ }^{1}$ Koji Onishi, ${ }^{2}$ \\ Yoshinori Kushiyama, ${ }^{3}$ Hirofumi Fujishiro, ${ }^{4}$ Youichi Miyaoka, ${ }^{4}$ Mika Yuki, ${ }^{5}$ \\ Yoshinori Komazawa, ${ }^{5}$ Takashi Tanimura, ${ }^{6}$ Hiroki Sonoyama, ${ }^{1}$ \\ Yasumasa Tada, ${ }^{1}$ Ryusaku Kusunoki, ${ }^{1}$ Akihiko Oka, ${ }^{1}$ Nobuhiko Fukuba, \\ Naoki Oshima, ${ }^{1}$ Ichiro Moriyama, ${ }^{7}$ and Yoshikazu Kinoshita ${ }^{1}$ \\ ${ }^{1}$ Department of Internal Medicine II, Shimane University School of Medicine, 89-1 Enya-cho, Izumo 6938501, Japan \\ ${ }^{2}$ Division of Internal Medicine, Matsue Seikyo Hospital, 8-8-8 Nishitsuda, Matsue 6908522, Japan \\ ${ }^{3}$ Division of Gastroenterology, Matsue Red Cross Hospital, 200 Horo-cho, Matsue 6908506, Japan \\ ${ }^{4}$ Division of Gastroenterology, Shimane Prefectural Central Hospital, 4-1-1 Himebara-cho, Izumo 6938553, Japan \\ ${ }^{5}$ Division of Internal Medicine, Izumo City General Medical Center, 613 Nadabunn-cho, Izumo 6910003, Japan \\ ${ }^{6}$ Division of Gastroenterology, Matsue City Hospital, 32-1 Noshira-cho, Matsue 6908509, Japan \\ ${ }^{7}$ Division of Cancer Center, Shimane University Hospital, 89-1 Enya-cho, Izumo 6938501, Japan
}

Correspondence should be addressed to Shunji Ishihara; si360405@med.shimane-u.ac.jp

Received 20 July 2014; Accepted 11 September 2014; Published 18 November 2014

Academic Editor: Toshimi Chiba

Copyright (C) 2014 Kousaku Kawashima et al. This is an open access article distributed under the Creative Commons Attribution License, which permits unrestricted use, distribution, and reproduction in any medium, provided the original work is properly cited.

\begin{abstract}
Purpose. Few reports have compared the clinical efficacy of a pH-dependent release formulation of mesalazine (pH-5-ASA) with a time-dependent release formulation (time-5-ASA). We examined whether $\mathrm{pH}$-5-ASA is effective for active ulcerative colitis (UC) in patients resistant to time-5-ASA. Methods. We retrospectively and prospectively analyzed the efficacy of pH-5-ASA in mildly to moderately active UC patients in whom time-5-ASA did not successfully induce or maintain remission. The clinical efficacy of pH-5-ASA was assessed by clinical activity index (CAI) before and after switching from time-5-ASA. In addition, the efficacy of $\mathrm{pH}-5$-ASA on mucosal healing $(\mathrm{MH})$ was evaluated in a prospective manner by measuring fecal calprotectin concentration. Results. Thirty patients were analyzed in a retrospective manner. CAI was significantly reduced at both 4 and 8 weeks after switching to $\mathrm{pH}-5-\mathrm{ASA}$. In the prospective study $(n=14)$, administration of $\mathrm{pH}-5$-ASA also significantly reduced CAI scores at 4 and 8 weeks in these patients who were resistant to time-5-ASA. In addition, fecal calprotectin concentration was significantly decreased along with improvement in CAI after switching to pH-5-ASA. Conclusions. Our results suggest that pH-5-ASA has clinical efficacy for mildly to moderately active patients with UC in whom time-5-ASA did not successfully induce or maintain remission.
\end{abstract}

\section{Introduction}

Mesalazine, a 5-aminosalicylic acid (5-ASA) compound, is widely recognized as the first line drug for induction therapy for mildly to moderately active ulcerative colitis (UC), because of its efficacy and safety [1-6]. Several formulations of oral mesalazine are available and primarily differentiated by the means of delivering active mesalazine to the colon. The time-dependent release formulation of mesalazine (time5-ASA) is coated with ethyl cellulose and begins to release 5-ASA in the duodenum; then the release is continued throughout the large intestine $[7,8]$. On the other hand, 
the $\mathrm{pH}$-dependent release formulation of mesalazine $(\mathrm{pH}-5-$ ASA) is coated with Eudragit-S and starts to release the drug in the terminal ileum or cecum, since the coating film breaks down at $\mathrm{pH} 7$ or higher $[9,10]$. High-dose treatments with these formulations are effective for induction of remission in patients with UC as compared to conventional dosages.

The therapeutic efficacy of oral mesalazine preparations for UC patients has been reported to be dependent on the efficiency of delivering active mesalazine to the colon. A recent study revealed that the mucosal mesalazine concentration in the sigmoid colon in patients treated with $\mathrm{pH}-5$-ASA was higher than that in patients treated with time-5-ASA [11]. Those findings suggest that $\mathrm{pH}-5$-ASA may be effective for active UC in patients for whom time-5-ASA did not successfully induce or maintain remission. However, it remains unknown whether switching from time-5-ASA to $\mathrm{pH}-5$-ASA contributes to therapeutic efficacy in those patients.

Mucosal healing $(\mathrm{MH})$ is currently regarded as an important treatment goal in patients with inflammatory bowel diseases (IBD) [12]. Although an endoscopy examination is recognized as the most reliable method for evaluating $\mathrm{MH}$ that is relatively invasive and sometimes painfull. Fecal calprotectin has been proposed as a noninvasive reliable surrogate marker for $\mathrm{MH}$, because of its strong correlation with endoscopically proven UC activity [13-15], and the clinical efficacy of fecal calprotectin for evaluating $\mathrm{MH}$ has been shown to be superior as compared to other noninvasive laboratory tests including erythrocyte sedimentation (ESR) and serum C-reactive protein (CRP) levels $[14,16]$.

The aim of this study was to investigate whether $\mathrm{pH}-5$ ASA is effective for mild to moderate UC in patients for whom time-5-ASA did not successfully induce or maintain remission. We performed retrospective and prospective studies to examine the effect of switching to $\mathrm{pH}-5$-ASA from time-5ASA to decrease clinical disease activity and induce $\mathrm{MH}$.

\section{Methods}

\subsection{Retrospective Study}

2.1.1. Patients. This retrospective study was conducted from January 2010 to August 2011 at 6 hospitals and 3 clinics in Japan. Eligible subjects were UC patients aged 18 years or older with a Rachmilewitz clinical activity index (CAI) [17] greater than 5 and/or rectal bleeding. In addition, the mesalazine formulation used in those patients was switched from time-5-ASA (Pentasa) to pH-5-ASA (Asacol) because of exacerbation or insufficient efficacy under administration of time-5-ASA (Pentasa) at greater than $2.25 \mathrm{~g} /$ day for at least 2 months. Patients were excluded if they had received oral salazosulfapyridine, corticosteroids, immunomodulatory drugs, or biologics or had undergone leukocytapheresis. Patient demographics, age, sex, disease extent, and doses of time-5ASA and pH-5-ASA were also investigated.

2.1.2. Study Design and Statistical Analysis. Change in CAI was retrospectively assessed at 0,4 , and 8 weeks after switching to $\mathrm{pH}-5$-ASA. The frequency of usage of a mesalazinebased enema during the prior 7 days in weeks 0 and 8 was also investigated. Clinical assessment was evaluated after 8 weeks as follows: remission: CAI 0 or 1, improvement: CAI decreased by more than 2 points, no change: $\mathrm{CAI}$ not changed or decreased by 1 point only, and exacerbation: CAI increased or increased in frequency of mesalazine-based enema use. Changes in CAI were statistically analyzed using Wilcoxon's signed rank test. $P<0.05$ was considered to be statistically significant.

2.2. Prospective Study. This open-label prospective study was conducted at Shimane University Hospital and Matsue Seikyo Hospital from August 2011 to July 2013. The ethics committee at each institution approved the protocol and all patients gave written informed consent in accordance with the Helsinki Declaration.

2.2.1. Patient Selection. UC patients aged 18 years and older with mild to moderate activity (CAI between 5 and 11) that was exacerbated during maintenance therapy using time5-ASA (Pentasa) greater than $2.25 \mathrm{~g}$ /day were enrolled as subjects. Exclusion criteria were as follows: treatment with oral salazosulfapyridine, corticosteroids, immunomodulatory drugs, or biologics for at least 3 months; receiving leukocytapheresis therapy; severe active UC (CAI 12 or more); positive results of stool culture for bacterial pathogens; current renal or hepatic disease; or medical contraindication for study participation. Patient demographics, age, sex, disease extent, duration and dose of time-5-ASA, and severity were investigated.

2.2.2. Study Schedule. In patients who met the inclusion criteria, $\mathrm{pH}$-5-ASA was administrated instead of time-5-ASA for 8 weeks. A daily dose of $2.25 \mathrm{~g}$ of time-5-ASA was switched to $2.4 \mathrm{~g}$ of pH-5-ASA, while a daily dose above $2.25 \mathrm{~g}$ of time5-ASA was switched to $3.6 \mathrm{~g}$ of $\mathrm{pH}-5-\mathrm{ASA}$. Patients using a mesalazine-based enema were allowed to continue that treatment at the same dosage and frequency during the study. Nonsteroidal anti-inflammatory drugs and antidiarrheal and antispasmodic medications were not allowed during the study.

In order to determine CAI, the frequency of bowel movements, bloody stools, and abdominal pain were monitored at weeks 0,4 , and 8. Peripheral blood samples were collected for measurement of complete blood count, ESR, and high sensitive CRP (hsCRP). Patients who required additional treatments based on physician assessment were withdrawn from the study at that time.

2.2.3. Measurement of Fecal Calprotectin. Fecal samples were collected twice at weeks 0 and 8 . In patients withdrawn from the study due to exacerbation, fecal samples were collected on the day of study discontinuation. Fecal samples were stored in a freezer at $-20^{\circ} \mathrm{C}$ until measurements. The calprotectin concentration was determined using a quantitative enzymelinked immunosorbent assay (PhiCal, Immundiagnostik, Germany). 
TABLE 1: Demographics of 30 eligible patients in retrospective study.

\begin{tabular}{lc}
\hline Age at entry (years) & $46.1 \pm 14.8$ \\
Gender (M/F) & $12 / 18$ \\
Disease extent & 5 \\
$\quad$ Extensive & 12 \\
Left-sided & 13 \\
Proctitis & \\
Dose of prior mesalazine (Pentasa) & 15 \\
$2250 \mathrm{mg}$ & 3 \\
$3000 \mathrm{mg}$ & 12 \\
$4000 \mathrm{mg}$ & \\
Dose of switched mesalazine (Asacol) & 12 \\
$2400 \mathrm{mg}$ & 18 \\
$3600 \mathrm{mg}$ & \\
\hline
\end{tabular}

2.2.4. Assessment and Statistical Analysis. The primary endpoint for the study was clinical efficacy after switching to $\mathrm{pH}$ 5-ASA treatment. Changes in CAI scores (at weeks 4 and 8) were statistically analyzed using Wilcoxon's signed rank test. Clinical assessment was evaluated at week 8 as follows: remission: CAI 0 or 1, improvement: CAI decreased by more than 2 points, no change: CAI not changed and decreased by 1 point only, and exacerbation: CAI increased. The secondary endpoint was a decrease in fecal calprotectin concentration. Changes in fecal calprotectin concentration were analyzed using Wilcoxon's singed rank test. $P<0.05$ was considered to be statistically significant.

\section{Results}

3.1. Retrospective Study. Thirty patients who met the inclusion criteria were enrolled and their demographics are shown in Table 1. The mean dose of time-5-ASA was $3025 \pm$ $839.1 \mathrm{mg} /$ day, while that of $\mathrm{pH}-5$-ASA after switching was $3120 \pm 597.9 \mathrm{mg} /$ day. Changes in mean CAI are presented in Figure 1(a). Mean CAI at week 0 was $5.20 \pm 1.84$, while that at weeks 4 and 8 was $2.73 \pm 2.27$ and $1.50 \pm 1.33$, respectively. CAI was significantly reduced at both weeks 4 and $8(P<0.001)$ after switching to $\mathrm{pH}-5$-ASA. Mean CAI in 12 patients who switched from time-5-ASA at $4 \mathrm{~g}$ /day was also significantly reduced (before, $5.08 \pm 1.31 ; 4$ weeks, $2.50 \pm 2.02 ; 8$ weeks, $1.58 \pm 1.08)$. Clinical assessment findings at week 8 are shown in Figure 1(b). Twenty-four patients $(80.0 \%)$ showed improvement or remission.

\subsection{Prospective Study}

3.2.1. Patient Characteristics. Fourteen patients who met the inclusion criteria were enrolled and their baseline characteristics are shown in Table 2. The mean age at entry was $45.1 \pm 16.6$ years old. The daily dose of time-5-ASA before switching to $\mathrm{pH}-5$-ASA was $2.25 \mathrm{~g}$ in 10 and $3.0 \mathrm{~g}$ in 4 patients. Clinical severity at entry was mildly active in 10 patients and moderately active in 4 patients. No patient had received a mesalazine-based enema prior to entry.
3.2.2. Clinical Efficacy. Of the 14 patients enrolled, 1 male (case 14) was excluded from analysis of efficacy because of insufficient compliance to the protocol. Therefore, 13 patients were analyzed for clinical efficacy. Of those, 11 continued the $\mathrm{pH}-5$-ASA administration for 8 weeks, while 2 patients (cases 9 and 10) were withdrawn from the study because their physicians decided that additional treatments were needed due to exacerbation at week 4 . Changes in CAI for each patient are presented in Figure 2. Mean CAI was $6.15 \pm 1.63$ at week $0,3.62 \pm 3.12$ at week 4 , and $1.82 \pm 1.40$ at week 8. CAI scores at weeks 4 and 8 were significantly reduced as compared to that at entry (week $4, P=0.009$; week 8 , $P=0.002)$. Clinical assessments at week 8 showed remission in 7 , improvement in 3 , no change in 1 , and exacerbation in 2 ; thus 10 patients $(76.9 \%)$ showed improvement or remission. No adverse effects were observed during this study.

\subsubsection{Changes in Fecal Calprotectin Concentration and Serum} hsCRP Level. Of the 13 patients analyzed, 1 (case 13) did not send in the fecal sample and 2 withdrew. Thus, the change in fecal calprotectin concentration at week 8 was analyzed in 10 patients. Changes in fecal calprotectin concentration for each patient are presented in Figure 3. Mean fecal calprotectin concentration at entry $(n=12)$ was $2288.6 \pm 3562.9 \mu \mathrm{g} / \mathrm{g}$ (median 1194.6), while that at week $8(n=10)$ was $395.6 \pm 581.3 \mu \mathrm{g} / \mathrm{g}$ (median 217.5). The fecal calprotectin concentration at week 8 was significantly reduced as compared to that at entry $(P=$ 0.012). Fecal samples from the 2 withdrawn patients (cases 9 and 10) were collected at week 4 and the fecal calprotectin concentration in that from case 9 was not reduced, while that from case 10 was slightly reduced. Mean hsCRP at entry was $0.459 \pm 0.576 \mathrm{~g} / \mathrm{L}$, while that at week 8 was $0.184 \pm 0.300 \mathrm{~g} / \mathrm{L}$. The decrease in hsCRP was not statistically significant $(P=$ 0.084).

\section{Discussion}

Results of both the retrospective and prospective studies clarified the therapeutic efficacy of switching to pH-5-ASA for mildly to moderately active UC patients in whom time5-ASA did not successfully induce or maintain remission. The therapeutic effect of $\mathrm{pH}-5-\mathrm{ASA}$ for $\mathrm{MH}$ was clearly shown by a decrease in fecal calprotectin concentration in the prospective study. Our results suggest that switching to $\mathrm{pH}$ 5-ASA is a viable therapeutic option for UC patients resistant to time-5-ASA.

Although dose-related efficacies of various oral mesalazine formulations for induction of remission in active UC patients have been reported [18-22], there are few direct comparisons of those based on the different systems for delivery of active mesalazine to the colon. A double-blind randomized trial that compared the efficacy of 2 different mesalazine formulations was performed in Japan by Ito et al. [23], which showed that $\mathrm{pH}-5-\mathrm{ASA}$ at $2.4 \mathrm{~g}$ /day was equally effective as time-5-ASA at $2.25 \mathrm{~g} /$ day for patients with active UC. However, to the best of our knowledge, there is no report regarding the clinical efficacy of $\mathrm{pH}-5$-ASA in active UC patients resistant to time-5-ASA. 


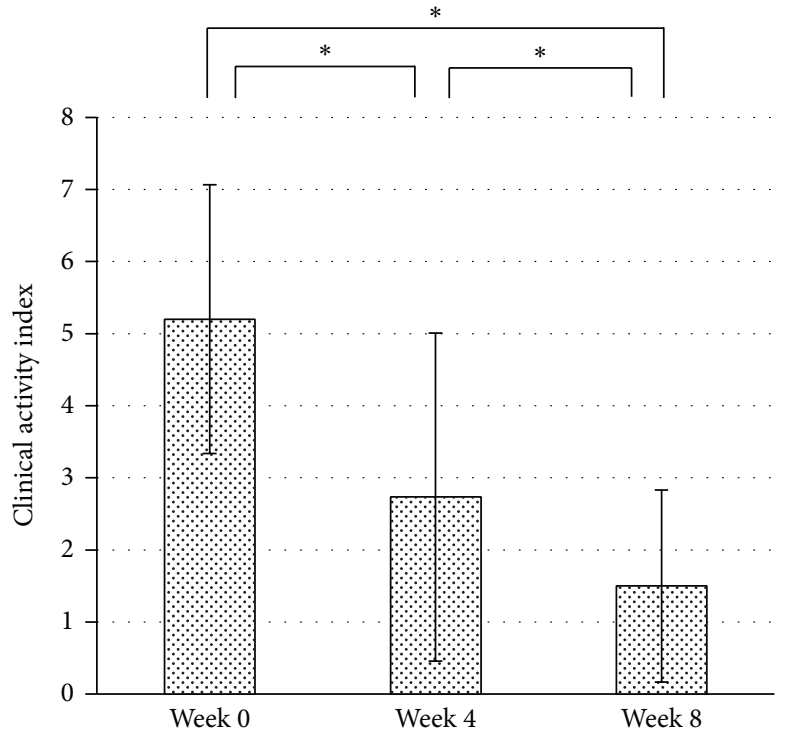

(a)

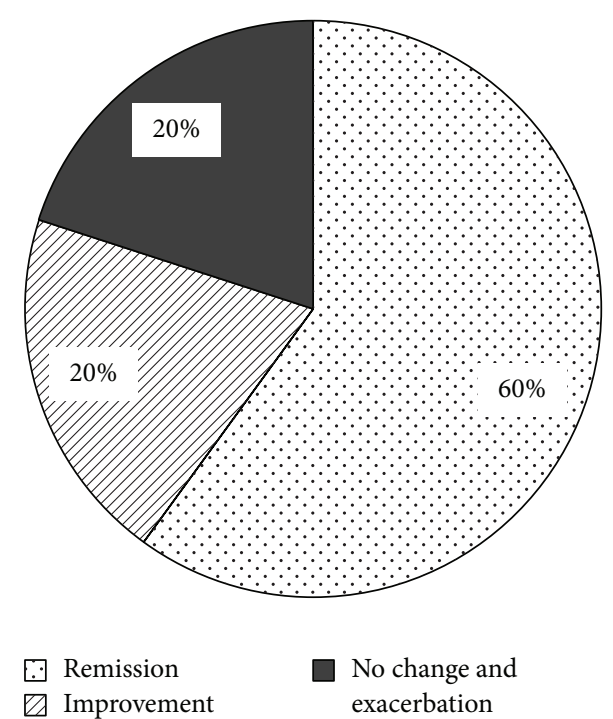

(b)

Figure 1: Results of retrospective study. (a) Changes in clinical activity index. (b) Clinical assessments at 8 weeks. ${ }^{*} P<0.001$.

TABLE 2: Baseline characteristics of 14 eligible patients in prospective study.

\begin{tabular}{|c|c|c|c|c|c|c|}
\hline Patient number & Age (years) & Gender & Disease extent & Dose of prior mesalazine (mg) & Severity & Rachmilewitz CAI \\
\hline 1 & 27 & $\mathrm{M}$ & Extensive & 3000 & Mild & 5 \\
\hline 2 & 73 & $\mathrm{~F}$ & Left-sided & 3000 & Mild & 6 \\
\hline 3 & 37 & M & Left-sided & 2250 & Mild & 5 \\
\hline 4 & 39 & $\mathrm{~F}$ & Extensive & 3000 & Mild & 5 \\
\hline 5 & 63 & $\mathrm{~F}$ & Extensive & 2250 & Moderate & 10 \\
\hline 6 & 33 & $\mathrm{~F}$ & Left-sided & 2250 & Mild & 5 \\
\hline 7 & 59 & $\mathrm{~F}$ & Extensive & 3000 & Mild & 6 \\
\hline 8 & 40 & M & Extensive & 2250 & Mild & 5 \\
\hline 9 & 27 & M & Extensive & 2250 & Moderate & 8 \\
\hline 10 & 57 & M & Left-sided & 2250 & Moderate & 7 \\
\hline 11 & 27 & $\mathrm{~F}$ & Proctitis & 2250 & Mild & 5 \\
\hline 12 & 72 & M & Extensive & 2250 & Moderate & 8 \\
\hline 13 & 31 & $\mathrm{~F}$ & Proctitis & 2250 & Mild & 5 \\
\hline 14 & 47 & M & Left-sided & 2250 & Mild & 5 \\
\hline
\end{tabular}

Oral mesalazine exerts its anti-inflammatory effect directly on inflamed mucosa in the large intestine, while therapeutic efficacy is thought to depend on colonic mucosal concentrations of the drug [24, 25]. Recently, D'Incà et al. showed that the mean mucosal concentration of mesalazine was significantly higher in $\mathrm{pH}-5$-ASA-treated patients as compared to time-5-ASA-treated patients [11]. In this regard, we speculated that $\mathrm{pH}-5$-ASA may be effective for UC patients who show resistance to time-5-ASA.

Initially, we retrospectively analyzed the efficacy of $\mathrm{pH}-$ 5-ASA in UC patients with mild to moderate activity in whom time-5-ASA did not successfully induce or maintain remission. Our findings revealed that an 8-week administration of pH-5-ASA significantly reduced CAI and improved clinical assessment regardless of the extent of UC. In addition, efficacy was also clearly shown in patients who switched from high-dose time-5-ASA (4 g/day). To confirm the results of our retrospective study, we then performed a prospective study to investigate the efficacy of pH-5-ASA in active UC patients resistant to time-5-ASA. The clinical efficacy of switching to $\mathrm{pH}-5-\mathrm{ASA}$ was also shown in those patients. Taken together, the present results suggest that the efficacy of mesalazine in a certain population of UC patients is dependent on the type of formulation, which might be associated with efficient delivery of active mesalazine to the colon. In this regard, we speculated that switching to $\mathrm{pH}$-5-ASA may be more effective in left-sided UC or proctitis cases. However, the efficacy noted with switching to $\mathrm{pH}-5$-ASA was not related to disease extent in our retrospective and prospective studies. In addition, we did not find an association between disease severity and 


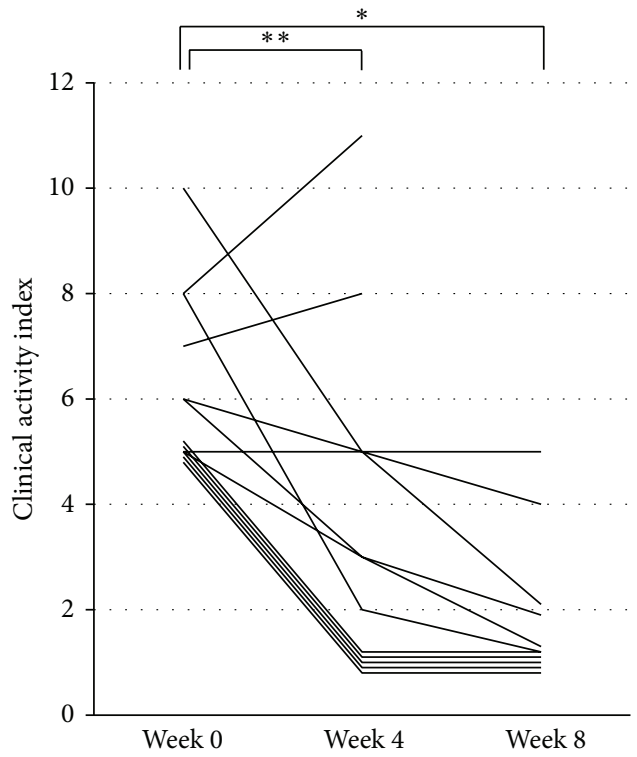

FIgURE 2: Changes in clinical activity index in prospective study. ${ }^{* *} P=0.009$ and ${ }^{*} P=0.002$.

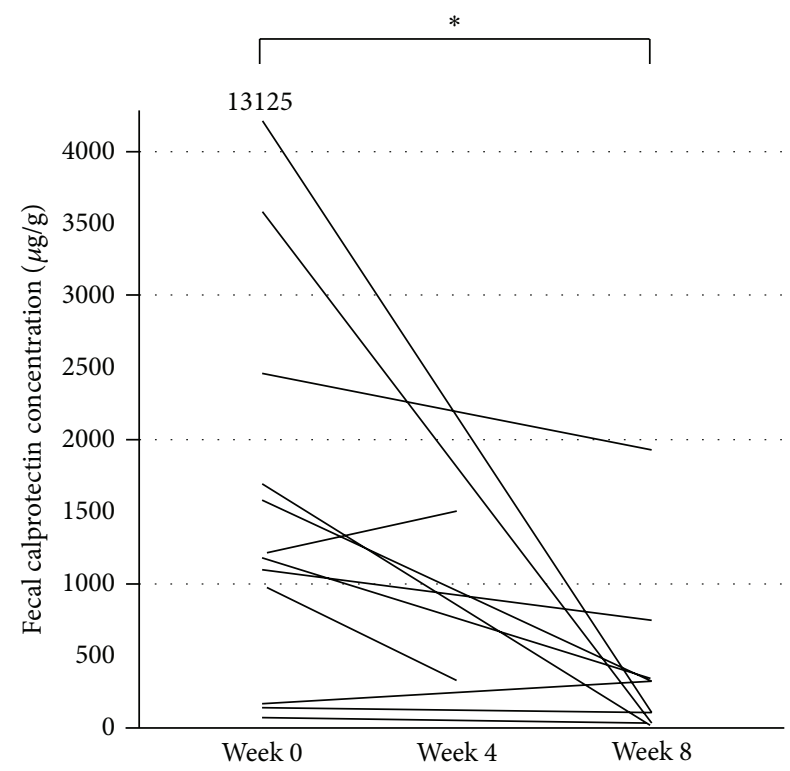

FIGURE 3: Changes in fecal calprotectin concentration in prospective study. ${ }^{*} P=0.012$.

efficacy after switching to $\mathrm{pH}-5$-ASA, though that result may be related to the small sample size.

Recently, MH has been proposed as a major therapeutic goal in clinical trials of UC patients [12] and has shown to be associated with higher clinical response and lower risk of clinical relapse [26-28]. A recent meta-analysis conducted by Römkens et al. demonstrated that an appropriate dose of oral mesalazine formulation successfully leads to $\mathrm{MH}$ along with induction of clinical remission in active UC patients [29]. In the present prospective study, we measured fecal calprotectin concentrations for evaluating the efficacy of $\mathrm{pH}-5-\mathrm{ASA}$ on $\mathrm{MH}$. Those results showed that $\mathrm{pH}-5-\mathrm{ASA}$ decreased fecal calprotectin concentration in UC patients who demonstrated exacerbation during maintenance therapy with time-5-ASA and suggested that switching to pH-5-ASA might have contributed to $\mathrm{MH}$ by efficiently delivering active mesalazine to inflamed mucosa. In contrast, serum hsCRP level was not significantly decreased in those patients after receiving $\mathrm{pH}$ 5-ASA treatment. Previous studies have demonstrated that endoscopically evident activity in UC is strongly correlated with fecal calprotectin concentration as compared to CRP $[14,30]$. Thus, CRP does not seem to be adequately sensitive for detecting low grade inflammation in assessment of $\mathrm{MH}$.

Our findings suggest that switching to $\mathrm{pH}-5-\mathrm{ASA}$ is a viable option as induction therapy for active UC patients who show resistance to time-5-ASA. However, it must be kept in mind that a delay of appropriate treatment may increase the severity of UC; thus attention must be given to determine whether an alternative treatment (e.g., corticosteroids) or switching to $\mathrm{pH}-5$-ASA should be used.

Our prospective study has several limitations. First, it was open label and there was no comparison made using a control group. A placebo effect may have existed in assessment of clinical efficacy. Second, the sample size was small. Third, selection bias may have occurred when we chose to enroll mildly to moderately active UC patients. In particular, disease severity in UC with moderate activity is distributed over a wide range. Finally, the concentration of mesalazine in colonic mucosa before and after switching the mesalazine formulation was not measured, though we speculate that the efficacy of pH-5-ASA may be dependent on efficient delivery of active mesalazine to the colon. To further confirm the efficacy of switching to another oral mesalazine formulation, a study considering these limitations should be conducted.

In conclusion, this is the first report to clarify the efficacy of $\mathrm{pH}-5$-ASA for UC patients with mild to moderate activity who show resistance to time-5-ASA. Furthermore, we revealed that $\mathrm{pH}-5-\mathrm{ASA}$ contributed to $\mathrm{MH}$ by confirming a decrease in fecal calprotectin concentration in those patients. Switching to $\mathrm{pH}-5$-ASA may be a viable therapeutic option for induction therapy in UC patients resistant to $\mathrm{pH}-5-\mathrm{ASA}$.

\section{Conflict of Interests}

The authors' institution received support from Zeria Pharmaceutical Co., Ltd., in Japan.

\section{References}

[1] G. R. Lichtenstein, M. A. Kamm, P. Boddu et al., "Effect of onceor twice-daily MMX mesalamine (SPD476) for the induction of remission of mild to moderately active ulcerative colitis," Clinical Gastroenterology and Hepatology, vol. 5, no. 1, pp. 95102, 2007.

[2] P. Marteau, C. S. Probert, S. Lindgren et al., "Combined oral and enema treatment with Pentasa (mesalazine) is superior to oral therapy alone in patients with extensive mild/moderate active ulcerative colitis: a randomised, double blind, placebo controlled study," Gut, vol. 54, no. 7, pp. 960-965, 2005. 
[3] G. D’Haens, D. Hommes, L. Engels et al., “Once daily MMX mesalazine for the treatment of mild-to-moderate ulcerative colitis: a phase II, dose-ranging study," Alimentary Pharmacology and Therapeutics, vol. 24, no. 7, pp. 1087-1097, 2006.

[4] R. Eliakim, Z. Tulassay, L. Kupcinskas et al., "Clinical trial: randomized-controlled clinical study comparing the efficacy and safety of a low-volume vs. a high-volume mesalazine foam in active distal ulcerative colitis," Alimentary Pharmacology and Therapeutics, vol. 26, no. 9, pp. 1237-1249, 2007.

[5] A. C. Moss and M. A. Peppercorn, "The risks and the benefits of mesalazine as a treatment for ulcerative colitis," Expert Opinion on Drug Safety, vol. 6, no. 2, pp. 99-107, 2007.

[6] B. G. Feagan, N. Chande, and J. K. MacDonald, "Are there any differences in the efficacy and safety of different formulations of oral 5-ASA used for induction and maintenance of remission in ulcerative colitis? Evidence from cochrane reviews," Inflammatory Bowel Diseases, vol. 19, no. 9, pp. 2031-2040, 2013.

[7] S. N. Rasmussen, S. Bondesen, E. F. Hvidberg et al., "5-Aminosalicylic acid in a slow-release preparation: bioavailability, plasma level, and excretion in humans," Gastroenterology, vol. 83, no. 5, pp. 1062-1070, 1982.

[8] R. N. Brogden and E. M. Sorkin, "A review of its pharmacodynamic and pharmacokinetic properties, and therapeutic potential in chronic inflammatory bowel disease," Drugs, vol. 38, no. 4, pp. 500-523, 1989.

[9] S. A. Riley, I. A. Tavares, A. Bennett, and V. Mani, "Delayedrelease mesalazine (5-aminosalicylic acid): coat dissolution and excretion in ileostomy subjects," British Journal of Clinical Pharmacology, vol. 26, no. 2, pp. 173-177, 1988.

[10] N. Q. Fernandez-Becker and A. C. Moss, "Improving delivery of aminosalicylates in ulcerative colitis: effect on patient outcomes," Drugs, vol. 68, no. 8, pp. 1089-1103, 2008.

[11] R. D'Incà, M. Paccagnella, R. Cardin et al., "5-ASA colonic mucosal concentrations resulting from different pharmaceutical formulations in ulcerative colitis," World Journal of Gastroenterology, vol. 19, no. 34, pp. 5665-5670, 2013.

[12] L. Peyrin-Biroulet, M. Ferrante, F. Magro et al., "Results from the 2nd Scientific Workshop of the ECCO (I): impact of mucosal healing on the course of inflammatory bowel disease," Journal of Crohn's and Colitis, vol. 5, no. 5, pp. 477-483, 2011.

[13] M. Daperno, F. Castiglione, L. de Ridder et al., "Results of the 2nd part Scientific Workshop of the ECCO (II): measures and markers of prediction to achieve, detect, and monitor intestinal healing in inflammatory bowel disease," Journal of Crohn's and Colitis, vol. 5, no. 5, pp. 484-498, 2011.

[14] A. M. Schoepfer, C. Beglinger, A. Straumann, M. Trummler, P. Renzulli, and F. Seibold, "Ulcerative colitis: correlation of the Rachmilewitz endoscopic activity index with fecal calprotectin , clinical activity, C-reactive protein, and blood leukocytes," Inflammatory Bowel Diseases, vol. 15, no. 12, pp. 1851-1858, 2009.

[15] G. D'Haens, M. Ferrante, S. Vermeire et al., "Fecal calprotectin is a surrogate marker for endoscopic lesions in inflammatory bowel disease," Inflammatory Bowel Diseases, vol. 18, no. 12, pp. 2218-2224, 2012.

[16] J.-Y. Xiang, Q. Ouyang, G.-D. Li, and N.-P. Xiao, "Clinical value of fecal calprotectin in determining disease activity of ulcerative colitis," World Journal of Gastroenterology, vol. 14, no. 1, pp. 5357, 2008.

[17] D. Rachmilewitz, F. Barbier, P. Defrance et al., "Coated mesalazine (5-aminosalicylic acid) versus sulphasalazine in the treatment of active ulcerative colitis: a randomised trial," British Medical Journal, vol. 298, no. 6666, pp. 82-86, 1989.
[18] N. Hiwatashi, Y. Suzuki, K. Mitsuyama, A. Munakata, and T. Hibi, "Clinical trial: effects of an oral preparation of mesalazine at $4 \mathrm{~g}$ /day on moderately active ulcerative colitis. A phase III parallel-dosing study," Journal of Gastroenterology, vol. 46, no. 1, pp. 46-56, 2011.

[19] T. Yamamoto, S. Umegae, and K. Matsumoto, "High-dose mesalazine treatment for ulcerative colitis patients who relapse under low-dose maintenance therapy," Digestive and Liver Disease, vol. 43, no. 5, pp. 386-390, 2011.

[20] S. B. Hanauer, W. J. Sandborn, C. Dallaire et al., "Delayedrelease oral mesalamine $4.8 \mathrm{~g} /$ day ( $800 \mathrm{mg}$ tablets) compared with $2.4 \mathrm{~g} /$ day ( $400 \mathrm{mg}$ tablets) for the treatment of mildly to moderately active ulcerative colitis: the ASCENDI trial," Canadian Journal of Gastroenterology, vol. 21, no. 12, pp. 827-834, 2007.

[21] W. J. Sandborn, J. Regula, B. G. Feagan et al., "Delayedrelease oral mesalamine $4.8 \mathrm{~g} /$ day ( 800 -mg tablet) is effective for patients with moderately active ulcerative coliti," Gastroenterology, vol. 137, no. 6, pp. 1934.e3-1943.e3, 2009.

[22] A. C. Ford, J.-P. Achkar, K. J. Khan et al., "Efficacy of 5-aminosalicylates in ulcerative colitis: systematic review and metaanalysis," The American Journal of Gastroenterology, vol. 106, no. 4, pp. 601-616, 2011.

[23] H. Ito, M. Iida, T. Matsumoto et al., "Direct comparison of two different mesalamine formulations for the induction of remission in patients with ulcerative colitis: a double-blind, randomized study," Inflammatory Bowel Diseases, vol. 16, no. 9, pp. 1567-1574, 2010.

[24] M. Naganuma, Y. Iwao, H. Ogata et al., "Measurement of colonic mucosal concentrations of 5-aminosalicylic acid is useful for estimating its therapeutic efficacy in distal ulcerative colitis: comparison of orally administered mesalamine and sulfasalazine," Inflammatory Bowel Diseases, vol. 7, no. 3, pp. 221-225, 2001.

[25] G. Frieri, R. Giacomelli, M. Pimpo et al., "Mucosal 5-aminosalicylic acid concentration inversely correlates with severity of colonic inflammation in patients with ulcerative colitis," Gut, vol. 47, no. 3, pp. 410-414, 2000.

[26] P. Rutgeerts, W. J. Sandborn, B. G. Feagan et al., "Infliximab for induction and maintenance therapy for ulcerative colitis," The New England Journal of Medicine, vol. 353, no. 23, pp. 24622476, 2005.

[27] J. F. Colombel, P. Rutgeerts, W. Reinisch et al., "Early mucosal healing with infliximab is associated with improved long-term clinical outcomes in ulcerative colitis," Gastroenterology, vol. 141, no. 4, pp. 1194-1201, 2011.

[28] G. Meucci, R. Fasoli, S. Saibeni et al., "Prognostic significance of endoscopic remission in patients with active ulcerative colitis treated with oral and topical mesalazine: a prospective, multicenter study," Inflammatory Bowel Diseases, vol. 18, no. 6, pp. 1006-1010, 2012.

[29] T. E. H. Römkens, M. T. Kampschreur, J. P. H. Drenth, M. G. H. van Oijen, and D. J. de Jong, "High mucosal healing rates in 5ASA-treated ulcerative colitis patients: results of a meta-analysis of clinical trials," Inflammatory Bowel Diseases, vol. 18, no. 11, pp. 2190-2198, 2012.

[30] J. Langhorst, S. Elsenbruch, J. Koelzer, A. Rueffer, A. Michalsen, and G. J. Dobos, "Noninvasive markers in the assessment of intestinal inflammation in inflammatory bowel diseases: performance of fecal lactoferrin, calprotectin, and PMN-elastase, CRP, and clinical indices," The American Journal of Gastroenterology, vol. 103, no. 1, pp. 162-169, 2008. 


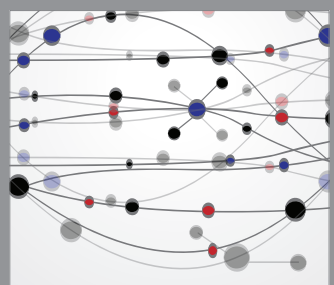

The Scientific World Journal
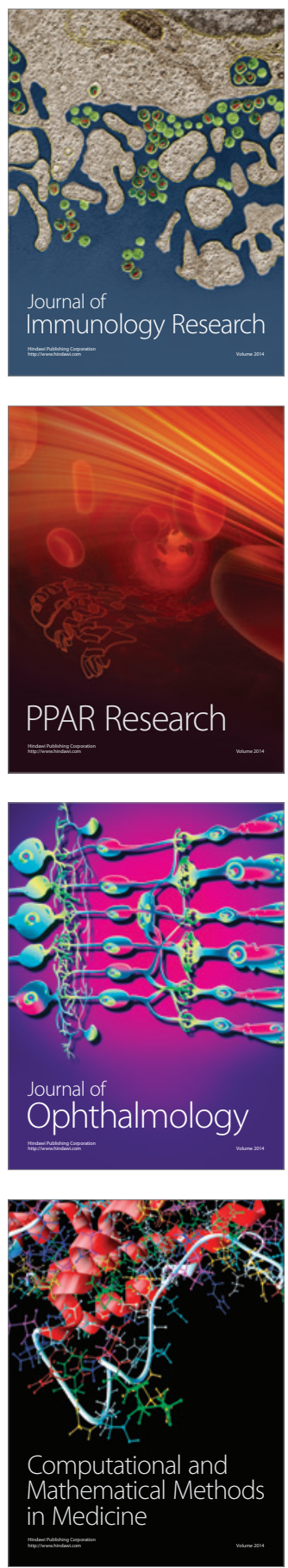

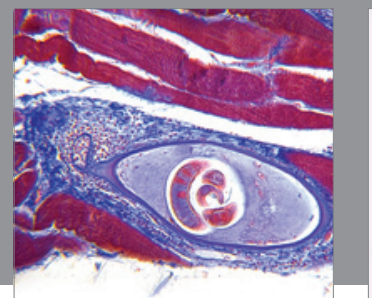

Gastroenterology

Research and Practice
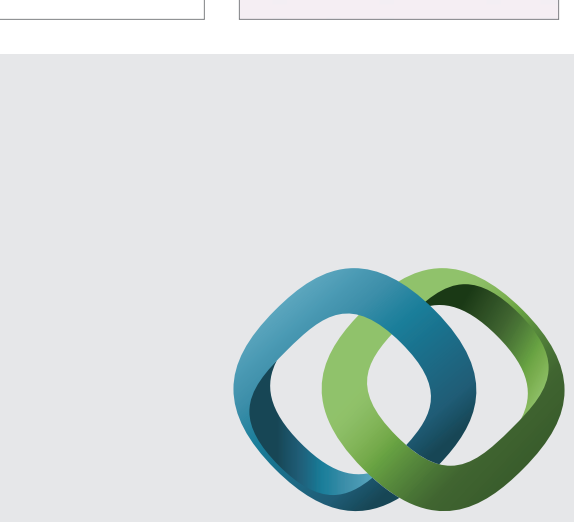

\section{Hindawi}

Submit your manuscripts at

http://www.hindawi.com
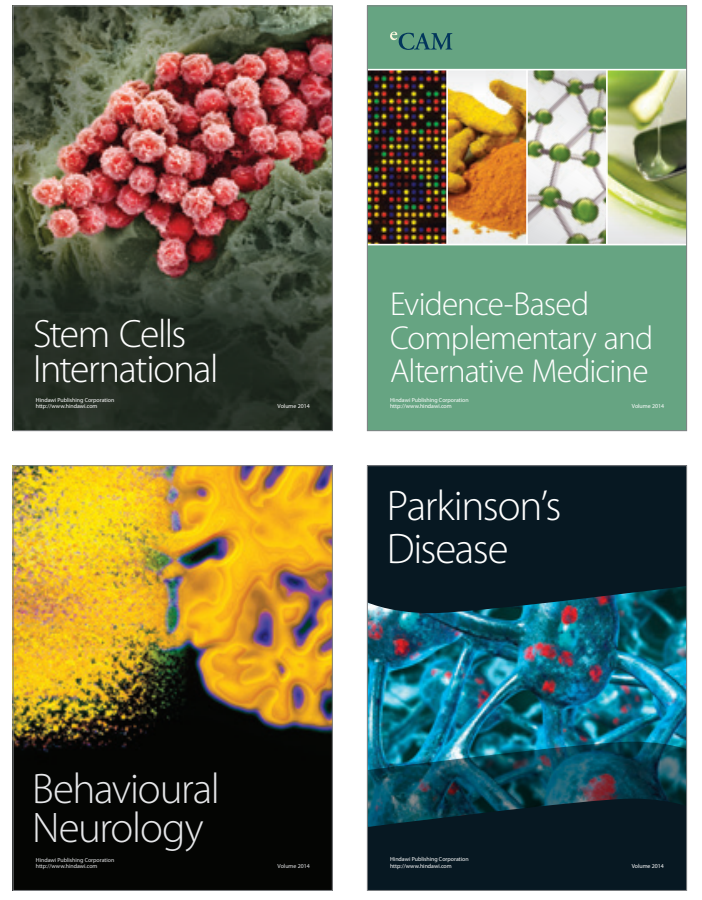
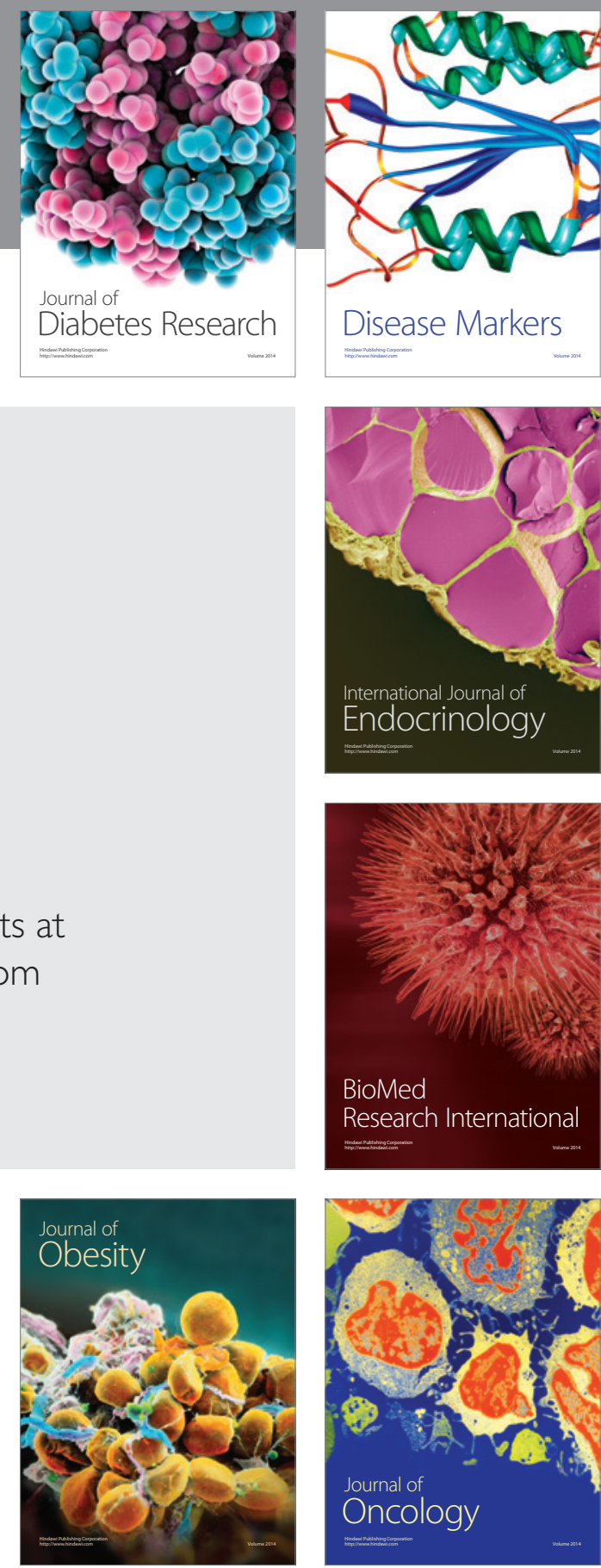

Disease Markers
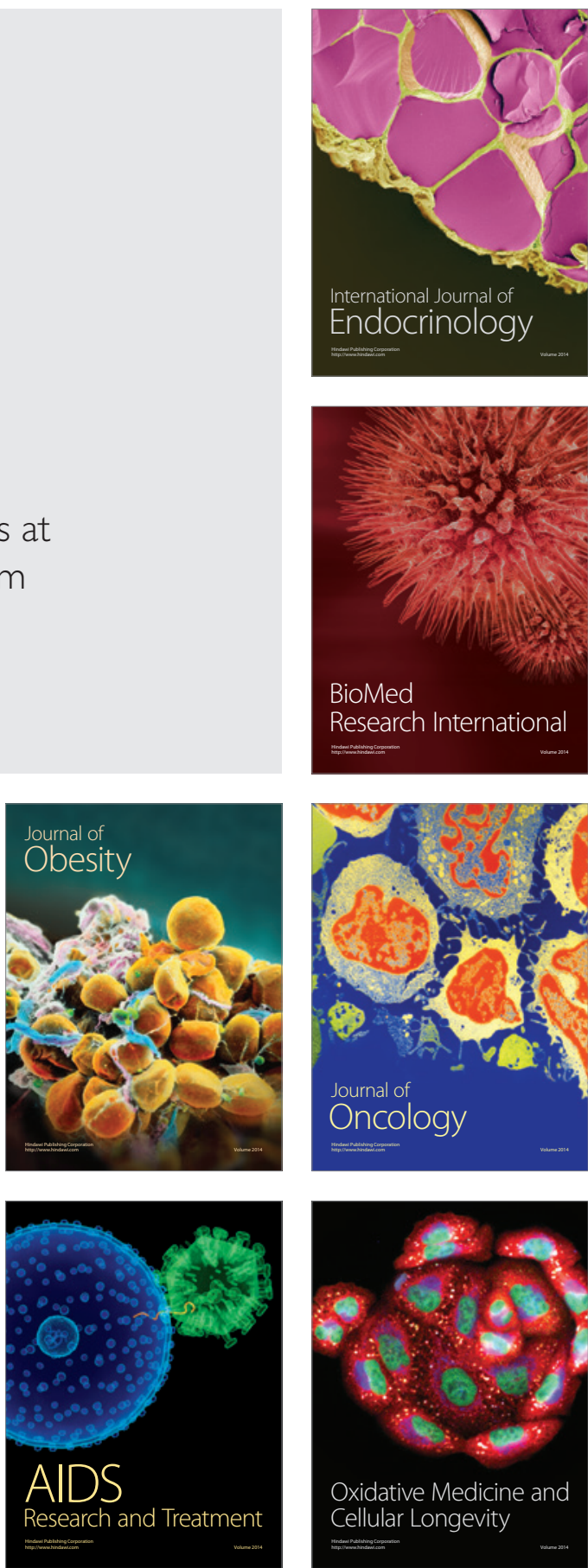